\title{
Metodologia para determinação da abundância de árvores urbanas utilizando recursos de geotecnologias de acesso livre
}

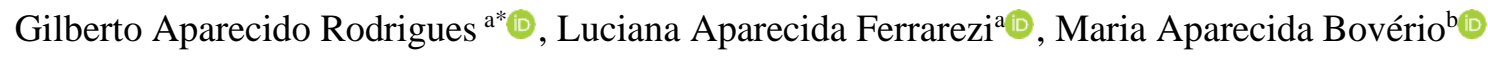 \\ ${ }^{a}$ Faculdade de Tecnologia de Taquaritinga, Brasil \\ ${ }^{\mathrm{b}}$ Faculdade de Tecnologia do Estado de São Paulo, Brasil \\ *Autor correspondente (gilberto.rodrigues@fatectq.edu.br)
}

\section{N F O}

Keywords
afforestation
learning
Google EarthPro
software
arboreal units

Palavras-chaves arborização aprendizagem sotware Google Earth Pro unidades arbóreas

\begin{abstract}
A B S T R A C T
Methodology for determining the abundance of urban trees using free access geotechnology resources The afforestation of different public spaces in cities guarantees several benefits for the urban ecosystem, among them, the improvement of the landscape, the guarantee of a favorable environment for the presence of the population of wild animals that live in these spaces, due to the restriction of natural environments and, in addition, it guarantees thermal comfort for the population. The objective of this study was to quantify the abundance of trees in an urban environment, using as a method the analysis of satellite images from the free geotechnology software Google Earth Pro. The results showed that the estimation of the number of trees in the city of Taiaçu, by counting trees on public roads detected by satellite images, it proved to be possible to use the technique by samples of urban public roads. It was also possible to determine 23 individual trees per kilometer, indicating that Taiaçu can be classified as a municipality that is next to the value considered critical. The use of the software proved to be easy to be executed with good precision for city of Taiaçu, SP.
\end{abstract}

\section{R E S U M O}

A arborização dos diferentes espaços públicos nas cidades garante vários benefícios para o ecossistema urbano, dentre eles estão a melhoria da paisagem, a garantia de ambiente favoravél para presença de populações de animais silvestres que convivem nesses espaços, devido à restrição dos ambientes naturais, além disso garante o conforto térmico para a população. $\mathrm{O}$ objetivo desse estudo foi o de quantificar a abundância arbórea em ambiente urbano, na cidade de Taiaçu, Estado de São Paulo - Brasil, utilizandose como método a análise de imagens de satélite, do software de geotecnologia livre Google Earth Pro. Os resultados mostraram que a estimativa do número de árvores no município, pela contagem de árvores em vias públicas avistadas por imagens de satélte, mostrou ser possível a utilização da técnica por amostragem de vias públicas urbanas. Foi possível determinar também o número de indivíduos arbóreos por kilômetro de 23 indicando-se que o município de Taiaçu pode ser enquadrado como um município pouco acima do valor considerado crítico. $\mathrm{O}$ uso do software mostrou ser de fácil execução e com boa precisão para o municipio de Taiaçu-SP-Brasil. 


\section{INTRODUÇÃO}

No Brasil, a arborização de ruas é uma atividade que passou a fazer parte do planejamento urbano, de forma generalizada, somente no final do século XIX, associada ao advento da República. No entanto, a partir da metade do século XX, o modelo de desenvolvimento gerou um processo de urbanização acelerado, e houve uma intensa ocupação dos espaços urbanos, com consequências na demanda de serviços de infraestrutura. Um conflito pela conquista desses espaços se dá, então, entre as árvores e os veículos, obras de construção e equipamentos públicos necessários para suprir a oferta crescente de serviços de infraestrutura e a preocupação com a qualidade ambiental (Meneghetti, 2003; Minaki e Amorim, 2007; Rossetti et al., 2010; Javed et al., 2017).

Duas grandes preocupações dos responsáveis pela arborização de uma cidade devem ser a de se atentar para a condição da planta no momento de ir a campo, e a sua altura até a primeira bifurcação do fuste, e também atentar-se com o tipo de poda a ser realizada futuramente para que as injúrias não conduzam à degradação das copas (Silva et al., 2019). A urbanização pode ser considerada uma das práticas mais homogeneizadoras dentre as principais atividades humanas. As cidades homogeneízam o ambiente físico, e como tal são construídas com o intuito de atender às necessidades relativamente restritas apenas da espécie humana. Nos espaços urbanos da periferia, muitas vezes, eles são ocupados por espécies nativas que se tornam regionalmente difundidas (Mckinney, 2005), diferentemente de espaços urbanos centrais, que são ocupados por uma classe social de maior poder aquisitivo.

A arborização é tema de preocupação em todas as partes do planeta, e como tal, na gestão dessa temática podem surgir muitos conflitos com a população em relação à convivência junto aos diferentes elementos da paisagem urbana (Emily et al., 2015). Dentre os elementos urbanos mais expressivos pode-se destacar a fachada dos imóveis, a natureza da via urbana, a largura da calçada, as placas de trânsito ou a disposição da rede elétrica (Randrup et al., 2001). Nesses aspectos, a grande demanda no manejo arbóreo, se não bem planejado pelo poder público, pode resultar em algum tipo de conflito com os usuários desses espaços urbanos, por exemplo, em função do grande número de árvores de grande porte, com mais de 9,0 m de altura, que de alguma forma pode interferir em algum tipo de elemento urbano (Pinheiro e Souza, 2017; Guimarães e Cardoso Júnior, 2019).

$\mathrm{O}$ tema arborização tem sido recentemente explorado e associado ao uso de geotecnologias, pelo fato de poder ser um instrumento de ensino que é capaz de contribuir na interiorização de conceitos de disciplinas de Geografia e outras ligadas ao ambiente, utilizando-se como principal ferramenta o software Google Earth (Sousa, 2018). Tal ferramenta propicia motivação e melhora nos resultados do ensino e da aprendizagem, através da mudança de percepção do aluno em relação aos assuntos ambientais, com destaque na importância do uso de geotecnologias no cotidiano escolar, tornando as aulas dinâmicas e mais eficientes na interiorização de conceitos geográficos. Nesse sentido, Silva e Chaves (2011), avaliaram a utilização do Google Maps e Google Earth no ensino médio, e foi possível constatar que os alunos se sentiram muito mais interessados em descobrir novas formas de aprendizado.

Oliveira e Lopes (2013), ao utilizarem softwares de geotecnologias (Google Maps) no ensino de geografia verificaram que tais tecnologias são ótimos instrumentos que proporcionam aulas mais dinâmicas, elevando o nível do conhecimento e proporciona uma nova abordagem ao conteúdo, principalmente, ao da disciplina de Geografia. Nessa mesma perspectiva, Souza e Costa (2017) afirmam que o uso dessa geotecnologia possibilitou uma visível contribuição em diversas disciplinas da educação, tais como a Geometria, a Física, em conceitos de recursos hídricos, e a aplicação do Google Earth no ensino propiciou um ambiente em sala de aula mais dinâmico e convidativo, devido a contextualização que aborda a realidade dos indivíduos.

Rachid e Couto (1999), compararam dois métodos de amostragem de árvores de rua afim de identificar qual é o mais eficiente para a cidade de São Carlos. Um método foi o levantamento quantitativo das árvores urbanas, empregando-se a amostragem casual simples e o outro método por amostragem estratificada por nível socioeconômico da população. O método de amostragem casual simples mostrou-se mais adequado para a determinação de número de árvores por quilômetro de via pública.

Meneghetti (2003), em um estudo para estimar a abundância de árvores no município de Santos-São Paulo-Brasil, desenvolvendo técnica semelhante proposta por Rachid e Couto (1999), constataram que o número de árvores por $\mathrm{km}^{2}$ foi de 38,93 indivíduos usando como unidade amostral um quarteirão. Assim, foi possível estimar os números de elementos arbóreos nos bairros com relativa precisão. Nessa mesma direção, Paiva (2009) também avaliou a arborização urbana na cidade paulista de Cosmópolis aplicando-se informações do número de árvores por quilômetro $(\mathrm{km})$ e utilizando o "quarteirão" como unidade amostral, semelhante aos estudos de Rachid e Couto (2009).

Iwama (2014) afirma, em seus estudos, que as pesquisas que consideram a determinação de arvores $/ \mathrm{km}$ de calçada, devem conceber como valores 
críticos para a quantificação de árvores em vias públicas o valor de 10 árvores $/ \mathrm{km}$. Cidades como Águas de São Pedro-SP-Brasil, e Jaboticabal-SPBrasil, são cidades que apresentam valores mais adequados, maiores ou igual a 100 unidades arbóreas $/ \mathrm{km}$ de calçada (Paiva, 2009; Paiva et al., 2010).

Silva e Sousa (2018), utilizando como instrumento de avaliação da arborização urbana a "Matriz de Leopold", verificaram que tal ferramenta permitiu diagnosticar de forma rápida e econômica, aspectos urbanos e ambientais afetados pela ausência da arborização. Silva et al. (2018), utilizando o Google Earth como ferramenta de estudo do espaço físico urbano, por meio de visitas in loco, constataram que apenas $6 \%$ da área do bairro era coberta por vegetação arbórea, sendo a estimativa de área verde por habitante de apenas $8,44 \%$. Os autores identificaram, ainda, 102 áreas livres públicas e 50 espécies arbóreas distintas. Dessas, apenas 24,5\% são espécies nativas e as demais são exóticas. A falta de arborização e a grande utilização de espécies exóticas sugerem afetar a presença e a permanência de diversos animais que se alimentam de flores e de frutos das espécies locais.

Guilherme et al. (2018), ao avaliarem a arborização em várias cidades do centro-oeste do Brasil, explicaram que $80 \%$ das mais de 3000 unidades arbóreas avaliadas foram plantadas em frente às residências, $14 \%$ em outros locais públicos, como igrejas, escolas e creches, e apenas $6 \%$ das árvores se concentraram em frente aos estabelecimentos comerciais, mas ficou evidente a falta de planejamento e controle do município na gestão dessa atividade em ambiente urbano. Explicaram, ainda, que $44 \%$ das avaliações ficavam fora da fiação e $56 \%$ ficavam sob fiação elétrica, sendo que $10 \%$ mostraram conflitos evidentes e apenas $22 \%$ das árvores compuseram-se por árvores exóticas.

Considerando-se todo o contexto de investigações realizadas sobre essa temática, o objetivo desse estudo foi o de estimar a quantidade de indivíduos arbóreos utilizando-se imagens de satélite do software livre Google Earth Pro.

\section{MATERIAL E MÉTODOS}

O município objeto de estudo foi a cidade de Taiaçu-São Paulo-Brasil, a qual dista da capital do estado 370 km, e 70 km de Ribeirão Preto-São Paulo - Brasil. Foi utilizado para esse estudo a análise de imagens de satélite por meio do software livre Google Earth Pro, inicialmente delimitando os quadrantes da cidade objeto de estudo, por duas linhas imaginárias fazendo-se a intersecção no centro da cidade, formando-se um ângulo de $90^{\circ}$ (Figura 1). Em seguida delimitou-se a área urbana de cada quadrante, utilizando-se a ferramenta "polígono". Posteriormente delimitou-se aleatoriamente a área amostral composta por 12 hectares (ha) em cada quadrante. Na sequência, e de forma aleatória, identificou-se três ruas na área amostral, em cada quadrante, por meio da ferramenta "caminho", a qual permite armazenar o comprimento de cada rua identificada e o número de elementos arbóreos identificados, através da contagem por observação visual da imagem, propiciada pelo software, vista de cima (Figura 2), num ângulo em relação ao solo de $90^{\circ}$, através da ferramenta de aproximação do distanciamento em relação ao solo, localizada do lado direito da área de trabalho do software aberto, como mostra a Figura 01, acionando o “ +" para aproximação, e "_" para afastamento.

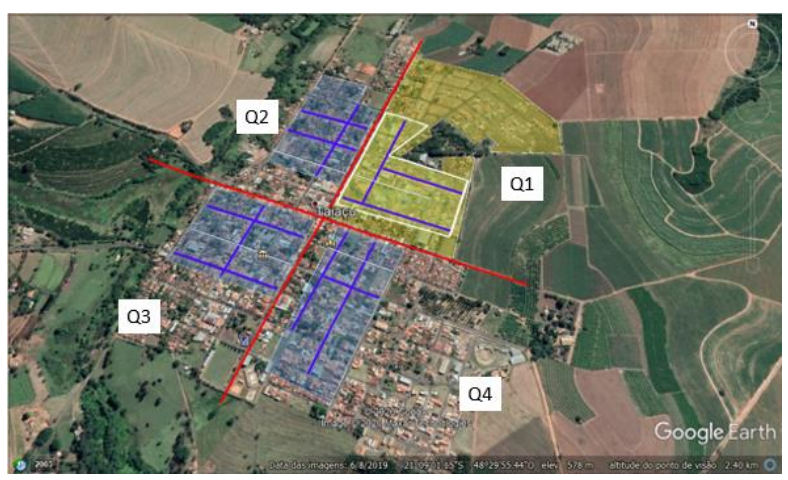

Figura 1 - Elementos básicos para visualização de imagens de satélite da cidade de Taiaçu-SP-Brasil, na identificação dos quadrantes (Q1, Q2, Q3 e Q4). O quadrante $1 \mathrm{em}$ amarelo identifica a área total urbana do quadrante, a linha tracejada branca denota a área amostral/quadrante, com as três vias públicas identificadas em azul. Nos outros quadrantes identifica-se apenas a camada selecionada da área amostral/quadrante com as três vias públicas.

Uma vez que cada caminho (via pública) tem seu comprimento verificado e armazenado no software, determinou-se os totais de comprimentos dos três caminhos (vias públicas) em cada quadrante (Tabela 1), assim como os totais do número de árvores desses três caminhos, em cada quadrante. Em seguida foi determinada a somatória de todos os comprimentos dos caminhos (vias públicas) em cada quadrante, utilizando-se a ferramenta "régua", a qual permite apenas a mensuração do comprimento da respectiva via pública.

Decidiu-se, então, de posse de todos os comprimentos das ruas de cada quadrante, a determinação da metragem de todas as vias urbanas do município. O comprimento de calçada total do município e o comprimento de calçada amostrada é o resultado da duplicidade do comprimento total das vias públicas do município, por se considerar os dois lados da calçada. Estabeleceu-se, então, o índice de 
árvores $/ \mathrm{km}$ que é resultado da razão entre o comprimento total das calçadas $(\mathrm{km})$ pelo número total de árvores no município. A notificação de indivíduos arbóreos amostrados por distância (m) é resultado da razão entre indivíduos arbóreos amostrados pelo comprimento de calçadas amostradas. Os dados foram organizados em quadrantes, comprimento das vias públicas observadas, número total de árvores observadas, área urbana de cada quadrante, comprimento das vias públicas amostradas e total de árvores amostradas e outras informações constantes na tabela 1 .

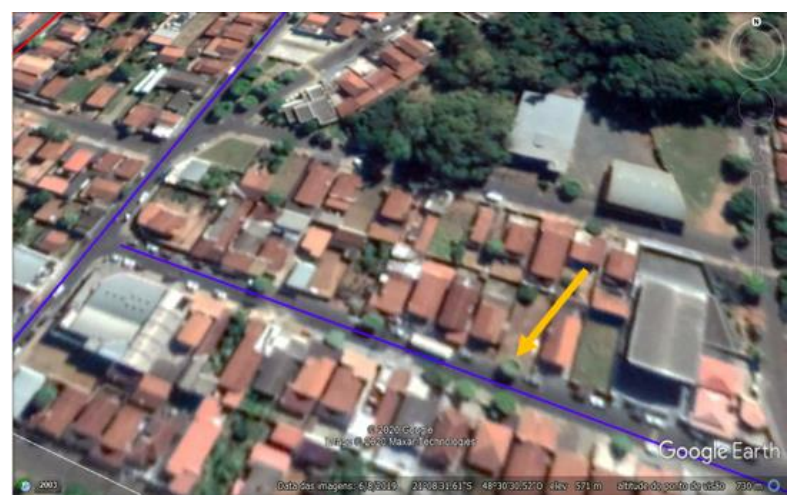

Figura 2 - Elementos básicos para observação de imagens de satélite de uma via pública da cidade de Taiaçu-SP-Brasil, na identificação da via pública e dos elementos arbóreos com distintos tamanhos (seta amarela).

Para a análise dos dados observados e amostrados constantes na tabela 1 , utilizou-se apenas o método comparativo entre os dados, e algumas relações de importância, tais como árvores/km e o distanciamento entre cada unidade arbórea. Para a proposta de estimativa das quantidades de árvores no município (EQAM) utilizou-se os dados da somatória de todos os comprimentos das vias públicas amostradas (SCVPA), as somatórias das áreas dos quadrantes amostrados (SAQA) (ha), o somatório de árvores amostradas em todos quadrantes (SAATQ) e a área total urbana (ATU) (ha), somandose a área total urbana de cada quadrante, excetuando-se os eventuais distritos, permite-se obter a medição do total das vias públicas (MTVP) (m):

$$
\text { MTVP }=\frac{(\text { SCVPA } \times \text { ATU })}{\text { SAQA }}
$$

Finalmente a EQAM é dada pala equação:

$$
\mathrm{EQAM}=\frac{(\text { MTVP } \times \text { SAATQ })}{\text { SCVPA }}
$$

Para os dados numéricos desse estudo para a cidade de Taiaçu-SP-Brasil, obteve-se os seguintes valores:

\author{
$\mathrm{SCVPA}=3432 \mathrm{~m}$ \\ MTVP $=9573,85 \mathrm{~m}$; \\ SAQA $=48$ ha; \\ SAATQ = 202 indivíduos arbóreos; \\ e ATU $=133,9$ ha
}

Portanto, EQAM = 563 unidades arbóreas estimadas. Para os dados estimados efetuou-se uma análise estatística univariada fazendo-se o uso do software livre Sisvar 5.6 (Ferreira, 2011), o qual foi aplicado o delineamento em blocos ao acaso, onde considerou-se cada área amostral por quadrante, como representativo de um bloco, e cada unidade caminho (via pública $=\mathrm{V}$ ), respectivamente $\mathrm{V} 1, \mathrm{~V} 2$ e V3, vias de cada quadrante, considerando-se como tratamento. Para efeito de controle local foi estabelecido um tamanho uniforme das unidades amostrais em cada quadrante de $12 \mathrm{ha}$, um número igualitário de vias públicas amostradas, e a casualidade na escolha da área amostral e das vias públicas. Aos dados foi aplicada a análise de variância e o teste de médias de Scott Knott a 5\% de probabilidade. Como hipótese desse estudo tem-se que: é possível estimar o número total de árvores no município amostrando apenas 12 vias públicas de maneira aleatória; o número de árvores/quilometro $(\mathrm{km})$ é considerado insatisfatório (menor ou igual a 10 unidades arbóreas $/ \mathrm{km}$ ); as unidades arbóreas por metro de calçada encontram-se a cada 10 metros.

\section{RESULTADOS E DISCUSSÃO}

Como resultados dos dados referentes às informações de comprimentos de vias públicas e números de árvores observadas e amostradas no município de Taiaçu-SP-Brasil, as informações da Tabela 1 mostram que as áreas amostradas nesse estudo corresponde a mais de $39 \%(48 / 121,9)$ da área do município.

Para confrontação da precisão dos dados estimados do número de árvores no município, dos dados dos comprimentos de todas as vias urbanas do município de Taiaçu-SP-Brasil, os quais perfazem um total de $24031 \mathrm{~m}$ de cada lado da calçada, realizouse a somatória de todas as árvores do município, de cada via pública, do número de unidades arbóreas observadas, 1073 unidades, e das unidades arbóreas estimadas, 563 unidades, e constatou-se pela razão entre esses valores, observadas /estimadas, que é possível ter um índice de correção, dada pela expressão:

Razão entre árvores observadas/estimadas $\frac{1073}{563}=$ 1,9 
Portanto, se EQAM = 563, o número aproximado de árvores no município é de 1,91x(EQAM).

Considerando-se que os indivíduos arbóreos / km estuda cada lado da calçada, é possível observar nos dados da tabela 1 que uma vez duplicando a metragem total das vias públicas $(24031 \mathrm{~m}=$
24,031 km), e dividindo-se pelo número de indivíduos arbóreos observados (1073), tem-se:

$$
\begin{aligned}
\text { Indiv. arbóreos } & =\frac{1070}{24,031 * 2} \\
= & 22,26 \text { ind } . \mathrm{km}^{-1}
\end{aligned}
$$

Tabela 1 - Dados referentes a informações de comprimentos de vias públicas e número de árvores observadas

\begin{tabular}{|c|c|c|c|c|c|}
\hline Quadrantes & $\begin{array}{l}\text { Comprimento vias } \\
\text { públicas observadas }\end{array}$ & $\begin{array}{c}\mathbf{N}^{0} \text { total de ár- } \\
\text { vores observa- } \\
\text { das }\end{array}$ & $\begin{array}{l}\text { Área urbana de } \\
\text { cada quadrante }\end{array}$ & $\begin{array}{c}\text { Comprimento } \\
\text { vias públicas } \\
\text { amostradas }\end{array}$ & $\begin{array}{l}\text { Total de árvo- } \\
\text { res amostradas }\end{array}$ \\
\hline $1^{\circ}$. & 7.223 & 264 & 34,4 & 1.069 & 63 \\
\hline $2^{\circ}$. & 3.396 & 179 & 25,0 & 279 & 20 \\
\hline $3^{\circ}$. & 5.015 & 272 & 25,0 & 1.035 & 36 \\
\hline $4^{\circ}$. & 8.397 & 358 & 37,5 & 1.049 & 43 \\
\hline Totais & 24.031 & 1.073 & 121,9 & 3.432 & 162 \\
\hline $\begin{array}{c}\text { Outras } \\
\text { informações }\end{array}$ & & $\begin{array}{l}\text { Área amostrada } \\
\text { Total de área am } \\
\text { Comprimento tot } \\
\text { omprimento das ca }\end{array}$ & $\begin{array}{l}\text { quadrante: } \\
\text { trada }(39 \%) \text { : } \\
\text { das calçadas } \\
\text { adas amostradas }\end{array}$ & & $\begin{array}{c}12 \mathrm{ha} \\
48 \mathrm{ha} \\
48.062 \mathrm{~m} \\
6.864 \mathrm{~m} \\
\end{array}$ \\
\hline
\end{tabular}
e amostradas no município de Taiaçu-SP-Brasil, utilizando-se o Google Earth Pro e outras informações.

$\mathrm{O}$ valor de 23 indivíduos arbóreos $/ \mathrm{km}$, segundo Iwama (2014), para o município de Taiaçu-SP-Brasil representa um valor pouco acima do valor crítico, considerado pelo número de 10 indivíduos arbóreos $/ \mathrm{km}$. Por outro lado, ao se considerar o mesmo raciocínio para os valores estimados dos comprimentos de vias amostradas $(3432 \mathrm{~m}=3,432 \mathrm{~km})$ e número de árvores amostradas (162), tem-se:

Indiv. arbóreos $=\frac{162}{3,432 * 2}=23,60 \mathrm{ind} \cdot \mathrm{km}^{-1}$

Quando se compara os indivíduos arbóreos/km e indivíduos arbóreos $/ \mathrm{km}$ estimados constata-se uma diferença de apenas 6\%. Quando se observa os resultados da análise de variância (Tabela 2), os dados demostraram não haver diferença significativa a 5\% entre o número de árvores estimadas em cada quadrante, quando em função das vias públicas amostradas. Semelhante observação pode ser feita com os dados da tabela 3 , em que não houve efeito significativo do comprimento das vias públicas em relação aos quadrantes avaliados. Não se constataram diferenças estatísticas entre as médias de números de árvores/quadrante, e no comprimento das vias/quadrante.

O que chama a atenção é que os coeficientes de variação foram elevados para as duas análises (Tabela 1 e 2), o que é plausível, pois não se consegue exercer controle local em relação a comprimento de vias públicas e número de árvores, apenas uniformiza-se a área a ser amostrada /quadrante, o número de vias a ser avaliada e a aleatoriedade na escolha dessas vias. É possível que, em relação aos valores dos coeficientes de variação elevados, eles podem ter influenciado na signifícância da análise de variância e do teste de médias. Quanto menor o coeficiente de variação mais homogeneidade espera-se no conjunto de dados. (Mohallem et al., 2008).

De acordo com o último censo do panorama das cidades do Instituto Brasileiro de Geografia e Estatística (IBGE, 2010; IBGE, 2015), o município de Taiaçu pertence ao bioma mata atlântica, tendo uma urbanização de vias públicas de 50,3\% e arborização de vias públicas de 74,8 \%. Pelas observações constatadas nesse estudo, o município de TaiaçuSP-Brasil situa-se pouco acima do índice crítico de árvores por km, com 22 indivíduos arbóreos. Entretanto, nesse estudo verificou-se 42 indivíduos arbóreos estimados $/ \mathrm{km}$, ou seja, um indivíduo arbóreo a cada 50 metros. As observações das imagens proporcionadas pelas imagens de satélite (Figura 2) mostram notoriamente que há muito espaço vazio nos dois lados das calçadas, onde poderia possivelmente ser ocupado por mais indivíduos arbóreos, podendo oferecer maior e melhor conforto térmico para a população (Pinheiro e Souza, 2017).

É possível que essa metodologia para cidades muito maiores possa ter suas limitações. Entretanto, o fato de poder-se estimar o número de árvores numa dada região por estimativa de menos de $10 \%$ no comprimento de vias públicas, facilita muito o trabalho das equipes de meio ambiente, 
e/ou equipes de poda de um dado município. Os resultados dessa pesquisa são promissores, mas devese constatar se o índice multiplicador desse estudo
$(1,91)$ se mantém o mesmo para municípios com maior número de habitantes.

Tabela 2 - Resumo da análise de variância da estimativa do número de árvores no município de Taiaçu-SP.

\begin{tabular}{l|c|c|c}
\hline Fonte de variação & GL & Fc & Prob $>\mathrm{Fc}$ \\
\hline Quadrantes & 3 & 0,716 & $0,5775^{\mathrm{ns}}$ \\
Vias públicas & 2 & 0,373 & $0,7034^{\mathrm{ns}}$ \\
\hline \multicolumn{5}{c}{ Média geral de número de arvores: 15,16} & CV: $(\%): 54,05$ \\
\hline \multicolumn{4}{c}{ Teste de médias } \\
\hline \multicolumn{5}{c}{ Valores médios por quadrantes do número de árvores estimadas } \\
\hline Q1 & Q2 & Q3 & Q4 \\
\hline 21,00a & $13,33 \mathrm{a}$ & $12.00 \mathrm{a}$ & $14.33 \mathrm{a}$
\end{tabular}

ns: indica que não houve efeito significativo a nível de 5\% probabilidade pelo teste F; Fc: F calculado (Snedecor); Prob: probabilidade; GL: grau de liberdade do teste; Letras minúsculas iguais na mesma linha indicam que não houve diferença significativa pelo teste de Scott Knott a 5\%; CV: Coeficiente de variação

Tabela 3 - Resumo da análise de variância do comprimento das vias públicas no município de Taiaçu-SP.

\begin{tabular}{l|c|c|c}
\hline Fonte de variação & GL & Fc & Prob > Fc \\
\hline Quadrantes & 3 & 0,301 & $0,8240^{\text {ns }}$ \\
Vias públicas & 2 & 1,283 & $0,3437^{\text {ns }}$ \\
\hline \multicolumn{4}{l}{ Média do comprimento das vias: 337m $\mathrm{CV}:(\%): 24,88$} \\
\hline \multicolumn{4}{c}{ Teste de médias } \\
\hline \multicolumn{4}{c}{ Valores médios por quadrantes do número de árvores estimadas } \\
\hline Q1 & Q2 & Q3 & Q4 \\
\hline 356,33a & $298,00 \mathrm{a}$ & $345,00 \mathrm{a}$ & $349,66 \mathrm{a}$ \\
\hline
\end{tabular}

ns: indica que não houve efeito significativo a nível de 5\% probabilidade pelo teste F; Fc: F calculado (Snedecor); Prob: probabilidade; GL: grau de liberdade do teste; Letras minúsculas iguais na mesma linha indicam que não houve diferença significativa pelo teste de Scott Knott a 5\%; CV: Coeficiente de variação.

O método proposto mostra-se, ainda, ser uma ferramenta de fácil execução e custo baixo. Pode ser utilizado por prefeituras ou empresas especializadas em serviços de poda nos municípios. Ao mesmo tempo, por envolver cálculos simples, pode até ser mais explorado em ambientes escolares para ser utilizada em disciplinas do ensino médio, para permitir maior interação com novas tecnologias e melhorar a aprendizagem (Silva e Chaves, 2011) assim como pode melhorar o grau de motivação dos alunos (Oliveira e Lopes, 2013) e desenvolver a percepção ambiental.

O uso de geotecnologias, tal qual o geoprocessamento e o sensoriamento remoto, pode ser uma importante ferramenta no monitoramento da arborização urbana, uma vez que a utilização da imagem de satélite de alta resolução mostra-se bastante eficiente na quantificação da cobertura vegetal (Lima e Vieira, 2009), e a associação com trabalho estruturado em campo amplia ainda mais as informações para o poder público local, no gerenciamento do ambiente em espaços urbanos, conforme os estudos bem estruturados de Guilherme et al. (2018), Silva et al. (2018) e Meneghetti (2003), que tiveram visitas in loco, e tal estratégia demanda um esforço e custo muito superior. Almeida et al. (2018) e Sampaio (2019), ressaltam que o uso do software livre Google Earth possui grande potencial para implantação do cadastro urbano em pequenos municípios e em outros serviços.

De maneira diferente, a proposta desse estudo delimitou-se em quantificar as unidades arbóreas, mas o software Google Earth Pro dispõe de ferramentas para visualização a nível de solo, de unidades arbóreas nas vias que são georreferenciadas (Google Street View), podendo facilitar em muito o trabalho de equipes ligadas à arborização urbana em um dado município, na vistoria da condição sanitária e até identificação das espécies arbóreas.

\section{CONCLUSÃO}

A determinação da abundância de unidades arbóreas pela amostragem de três vias públicas por quadrante foi possível e mostrou-se satisfatória para facilitar a gestão e o planejamento da arborização do município de Taiaçu-SP-Brasil. O número de árvores estimadas pelo método de amostragem por quadrante foi de 563 árvores no município, devendo-se usar um fator de correção de 1,91 para aproximar-se à contagem total de fato de 1070 
árvores. O índice de indivíduos arbóreos/km determinado para a cidade de Taiaçu-SP-Brasil foi de 23, valor esse considerado pouco adequado. $\mathrm{O}$ uso de software livre Google Earth Pro possui ferramentas capazes de dar suporte aos departamentos de planejamento urbano de cidades de pequena população.

\section{AGRADECIMENTOS}

Agradecimento especial à Diretoria da Faculdade de Tecnologia de Taquaritinga (Fatectq) e a CPRJI do CEETEPS, sem os quais o presente trabalho não poderia ter sido realizado.

\section{REFERÊNCIAS BIBLIOGRÁFICAS}

Emily EO, Robert JW, Alexander JF, Mark AB. Challenges and future directions in urban afforestation Journal of Applied Ecology, 2013.

http://dx.doi:10.1111/1365-2664.12124

Ferreira DF. SISVAR: um programa para análises e ensino de estatística. Revista Científica Symposium, Lavras, v.6, n.2, p.36-41, 2008 .

Guilherme FAG, Silva MC, Carneiro DNM, Nascimento HCA, Ressel K, Ferreira WC. Urban arborization in public pathways of four cities in east Mato Grosso do Sul (MS) Brazil. Ornamental horticulture, v.24, n.2, p.174-181, 2018.

http://dx.doi.org/10.14295/oh.v24i2.1137

Guimarães MG, Cardoso Júnior RAF. Diagnóstico e análise dos conflitos da arborização urbana: estudo de caso do bairro do Grajaú (Rio de Janeiro). Revista Internacional de Ciências, v.09, n.01, p.92-104, jan-abr,2019. http://dx.doi.org/10.12957/ric.2019.37464

Instituto Brasileiro de Geografia e Estatística (IBGE). Características urbanísticas do entorno dos domicílios. Censo Demográfico 2010. Censo demogr., Rio de Janeiro, p.1175,2010 .

Instituto Brasileiro de Geografia e Estatística (IBGE) - Indicadores de desenvolvimento sustentável: Brasil: 2015/IBGE, Coordenação de Recursos Naturais e Estudos Ambientais [e] Coordenação de Geografia. - Rio de Janeiro: IBGE, 2015. 352p. - (Estudos e pesquisas. Informação geográfica, ISSN 1517-1450; n.10.

Iwama AY. Indicador de arborização urbana como apoio ao planejamento de cidades brasileiras. REVSBAU, Piracicaba - SP, v.9, n.3, p.156-172, 2014. http://dx.doi.org/10.5380/revsbau.v9i3.63121

Javed T, Kamal K, Mathavan S Identifying tree species from google earth imagery: Detection of Palmyra trees in northern Sri Lanka. In: Proceedings of the 23rd International Conference on Automation e Computing, University of Huddersfield, Huddersfield, UK, 7-8 September, 2017.

Lima $\mathrm{M}$ de $\mathrm{O}$, Vieira $\mathrm{V}$ de CB.Uso de geotecnologias para análises da cobertura vegetal urbana. In: Anais... XIV Simpósio Brasileiro de Sensoriamento Remoto, Natal, Brasil, 25-30 abril 2009, INPE, p.731-738.
Mckinney ML. Urbanization as a major cause of biotic homogenization. Biological Conservation, v.127, p.247-260, 2006.

http://dx.doi.org/10.1016/j.biocon.2005.09.005

Meneghetti GIP Estudo de dois métodos de amostragem para inventário da arborização de ruas dos bairros da orla marítima do município de Santos, SP.,2003, 100 p. Dissertação (mestrado), Escola Superior de Agricultura Luiz de Queiroz, 2003. Piracicaba, 2003.100 p.

Minaki C, Amorim M C de CT. Espaços urbanos e qualidade ambiental - um enfoque da paisagem. Revista Formação, n.14, v.1, p.67-82.

Mohallem DF, Tavares M, Silva PL, Guimarães EC, Freitas RF. Avaliação do coeficiente de variação como medida da precisão em experimentos com frangos de corte. Arq. Bras. Med. Vet. Zootec., v.60, n.2, p.449-453, 2008. https://doi.org/10.1590/S0102-09352008000200026

Oliveira IN de, Lopes MC. O uso das novas tecnologias no ensino da geografia: Google Maps, Flightrader 24 e Marine Traffic abordando os meios de transporte aéreo e marítimo. In: Os desafios da escola pública paranaense na perspectiva do professor PDE, Cadernos PDE, v.1, 2013.

Paiva AV. Aspectos da Arborização Urbana do Centro de Cosmópolis-SP. Revista SBAU, v.4, n.4, p.17-31, 2009.

Paiva AV, Lima ABM, Carvalho A, Junior AM, Gomes A, Melo CS. Inventário e Diagnóstico da Arborização Urbana Viária de Rio Branco, AC. Revista SBAU, v.5, n.1, p.144$159,2010$.

Pinheiro CR, Souza D de. A importância da arborização nas cidades e sua influência no microclima. R. gest. sust. ambient., v.6, n.1, p.67-82, 2017.

https://doi.org/10.19177/rgsa.v6e1201767-82

Rachid C, Couto HTZ do. Estudo da eficiência de dois métodos de amostragem de árvores de rua na cidade de São Carlos - SP. Scientia Florestais, n.56, p.59-68, 1999.

Randrup TB, Mcpherson EG, Costello LRA. Review of tree root conflicts with sidewalks, curbs, and roads. Urban Ecosystems, v.5, p.209-225, 2001. https://doi.org/10.1023/A:1024046004731

Rossetti AIN, Pellegrino PRM, Tavares AR. Árvores e suas interfaces no ambiente urbano. REVSBAU, Piracicaba SP, v.5, n.1, p.1-24, 2010.

Silva ADP da, Batista AC, Giongo M, Biondi IDB, Santos AF dos, Cachoeira JN, Machado IES Aspectos qualitativos e quantitativos da arborização das praças públicas de $\mathrm{Gu}-$ rupi, TO, Brasil. Journal of Biotechnology and Biodiversity, v.6, n.1, 2018. https://doi.org/10.20873/jbb.uft.cemaf.

Silva ESC, Alencar PM, Silva RG da, Melo YL. Categorização dos espaços livres públicos e arborização em Neópolis: quais espécies podem ser encontradas no bairro?. Revista Cultural e Científica, UNIFACEX. v.16, n.2, 2018.

Silva ST da, Sousa BH de. Diagnóstico da arborização urbana do município de Guarabira-Paraíba. Paisag. Ambiente: ensaios. n.41, p.167-184, 2018. https://doi.org/10.11606/issn.2359-5361.v0i41p167-184. 